\title{
Derivatives of Cardanol through the Ene Reaction with Diethyl Azodicarboxylate
}

\author{
Atanu Biswas, ${ }^{*, a}$ Carlucio R. Alves, ${ }^{b}$ Maria T. S. Trevisan, ${ }^{c}$ Janet Berfield, ${ }^{a}$ \\ Roselayne F. Furtado, ${ }^{d}$ Zengshe Liu ${ }^{a}$ and Huai N. Cheng ${ }^{*, e}$ \\ ${ }^{a}$ USDA Agricultural Research Service, National Center for Agricultural Utilization Research, \\ 1815 North University Street, 61604, Peoria-IL, USA \\ ${ }^{b}$ Departamento de Química, Universidade Estadual do Ceará, Avenida Silas Munguba, 1700, \\ Campus Itaperi, 60740-020 Fortaleza-CE, Brazil \\ 'Departamento de Química, Universidade Federal do Ceará, Campus Pici, CP 6021, \\ 60455-760 Fortaleza-CE, Brazil \\ ${ }^{d}$ Embrapa Agroindústria Tropical, Rua Dra. Sara Mesquita, 2270, Planalto Pici, \\ 60511-110 Fortaleza-CE, Brazil \\ ${ }^{e}$ USDA Agricultural Research Service, Southern Regional Research Center, \\ 1100 Robert E. Lee Blvd., 70124, New Orleans-LA, USA
}

\begin{abstract}
Cardanol is an alkyl/alkenyl phenolic material obtained from cashew nut shell liquid (CNSL), which is a byproduct of cashew nut processing. In an effort to develop new uses, cardanol was derivatized for the first time with diethyl azodicarboxylate (DEAD) through the ene reaction. The reaction was facile and required only the application of heat without a catalyst. Both conventional heating and microwave heating were shown to be effective; the latter entailed much shorter reaction time and substantial energy savings. The reaction product (a hydrazino-ester derivative of cardanol) was characterized by nuclear magnetic resonance (NMR). The product increased in viscosity with time and may be useful as a viscosifier in oil-based commercial formulations and as a synthon for further organic reactions.
\end{abstract}

Keywords: cardanol, cashew nut shell liquid, diethyl azodicarboxylate, ene reaction

\section{Introduction}

Cashew nut shell liquid (CNSL) is a commercially available agricultural renewable resource and a byproduct of the cashew industry. Cardanol, obtained by double vacuum distillation of CNSL, is not only cheap but biodegradable and abundantly available, especially in India. ${ }^{1,2}$ It is an alkyl/alkenyl phenol where the non-aromatic chain contains 15 carbon atoms, which may be a saturated hydrocarbon (5-8\%), a mono-ene (48-49\%), a diene (16-17\%), or a triene (29-30\%) as shown in Figure 1.

Both the phenolic $\mathrm{OH}$ and the double bonds on the alkenyl chain are capable of chemical modifications. Indeed a large number of cardanol derivatives and applications have been published in recent years, including

*e-mail: atanu.biswas@ars.usda.gov, hn.cheng@ars.usda.gov

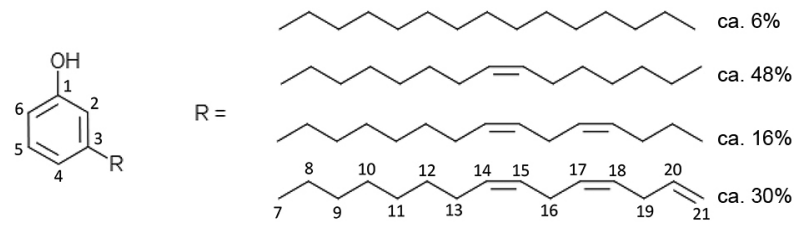

Figure 1. Schematic structure of cardanol.

phenolic resins from cardanol and formaldehyde, ${ }^{3-5}$ epoxy curing resins, ${ }^{6-8}$ flame retardants, ${ }^{9,10}$ polyurethanes, ${ }^{11-14}$ coatings, ${ }^{15-18}$ biobased polymers, ${ }^{19}$ and reactive diluents. ${ }^{20,21}$ More derivatives have been given in a recent review. ${ }^{1}$ Despite the voluminous literature, there seems to be no prior report on the use of ene reaction to derivatize the olefins on the alkenyl moieties of cardanol.

In the ene reaction, an electron-deficient olefin forms a bond with an olefin that contains an allylic hydrogen. ${ }^{22-24}$ It represents a group transfer pericyclic reaction and is related to Diels-Alder reaction. Because of its simplicity, it 
is a useful tool for the synthesis of complex molecules. ${ }^{22,23}$ Diethyl azodicarboxylate (DEAD) has been used in ene reactions, e.g., for soybean oil and DEAD. ${ }^{25,26}$ The purpose of this work is to explore the ene reaction with DEAD as a means to generate new derivatives from cardanol.

\section{Experimental}

Materials

Cardanol (stabilized, 88.5\%) was purchased from Shanghai Meidong Biological Material Co., Ltd. (Shanghai, China) and used after distillation at $240-250{ }^{\circ} \mathrm{C}$ under 3-5 Torr. DEAD, ethyl acetate and deuterochloroform were acquired from Sigma Aldrich (Milwaukee, Wisconsin, USA) and were used without further purification.

\section{Reaction of cardanol and DEAD}

In the case of conventional heat, typically $0.257 \mathrm{~g}$ cardanol, 0.090-0.175 g DEAD, and $1 \mathrm{~mL}$ ethyl acetate were mixed in a vial. The vial was then stirred in a Reacti-therm ${ }^{\mathrm{TM}}$ set at $70{ }^{\circ} \mathrm{C}$ for 6 hours at atmospheric pressure. The yellow color of the reaction mixture slowly disappeared and became colorless. When the reaction was completed, the reaction mixture was placed in a vacuum oven overnight where the ethyl acetate was evaporated.

The microwave-assisted reaction was conducted at atmospheric pressure on a Biotage $\AA$ Initiator Microwave Synthesis Systems (Biotage AB, Uppsala, Sweden). For each reaction $0.257 \mathrm{~g}$ cardanol, 0.090-0.175 g DEAD, and $1 \mathrm{~mL}$ ethyl acetate were placed in a Biotage reaction vial with stir bar and cap. The temperature was set at $70{ }^{\circ} \mathrm{C}$, and microwave energy was applied to hold the temperature at $70{ }^{\circ} \mathrm{C}$ for 5 minutes. The cap was removed, and the reaction mixture transferred to a $20 \mathrm{~mL}$ glass scintillation vial. It was first evaporated in a rotary evaporator and then placed in $50{ }^{\circ} \mathrm{C}$ vacuum oven overnight to remove ethyl acetate.

\section{Nuclear magnetic resonance (NMR) spectroscopy}

All nuclear magnetic resonance (NMR) spectra were acquired with a Bruker ARX-500 spectrometer (Bruker, Rheinstetten, Germany) at ${ }^{1} \mathrm{H}$ frequency of $500 \mathrm{MHz},{ }^{13} \mathrm{C}$ frequency of $125 \mathrm{MHz}$, and a 5-mm dual probe. The sample solutions were prepared in deuterochloroform $\left(\mathrm{CDCl}_{3}\right.$, 99.8\% D, Cambridge Isotope Laboratories, Inc., Andover, MA, USA). Standard operating conditions were used.

\section{Viscosity}

Viscosity measurements were conducted on a TA Instruments (New Castle, Delaware, USA) LS1 controlledstrain fluids rheometer. All measurements were performed at $25^{\circ} \mathrm{C}$ using a peltier plate system with a $25 \mathrm{~mm}$ titanium parallel plate. Rate sweeps were performed from 0.1 to $100 \mathrm{~s}^{-1}$. TRIOS software (version 3.3.1.4055) was used to calculate viscosity, assuming a Newtonian fit.

\section{Results and Discussion}

In the ene reaction, DEAD served as the electron deficient olefin, and the allyl hydrogen was provided by the alkenyl moiety on cardanol (Scheme 1).

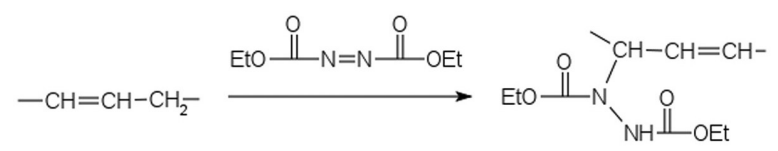

Scheme 1. Reaction of DEAD with an olefin on the alkenyl moiety of cardanol.

The ene reaction was carried out by mixing cardanol and DEAD with ethyl acetate as a solvent. In the conventional heat reaction, the mixture was heated and stirred at $70^{\circ} \mathrm{C}$ for 6 hours. For the microwave-assisted reaction, the mixture was heated and stirred at $70{ }^{\circ} \mathrm{C}$ for 5 minutes. The ethyl acetate was then removed and the reaction mixture analyzed. Some typical reactions are summarized in Table 1.

Table 1. Typical reactions of cardanol with DEAD, conducted with $1 \mathrm{~mL}$ of ethyl acetate

\begin{tabular}{|c|c|c|c|c|c|}
\hline Sample & Weight of cardanol / $g$ & Weight of DEAD / g & $\begin{array}{c}\text { DEAD }^{\text {a }} \text { per cardanol, } \\
\text { weight ratio }\end{array}$ & Reaction mode & Reaction condition \\
\hline $\mathrm{C}-0$ & 0.255 & 0 & 0 & none & \\
\hline $\mathrm{C}-1$ & 0.258 & 0.090 & 0.349 & conventional heat & $70^{\circ} \mathrm{C}, 6 \mathrm{~h}$ \\
\hline $\mathrm{C}-2$ & 0.253 & 0.175 & 0.692 & conventional heat & $70^{\circ} \mathrm{C}, 6 \mathrm{~h}$ \\
\hline M-1 & 0.252 & 0.090 & 0.357 & microwave & $70^{\circ} \mathrm{C}, 5 \mathrm{~min}$ \\
\hline M-2 & 0.252 & 0.175 & 0.595 & microwave & $70^{\circ} \mathrm{C}, 5 \mathrm{~min}$ \\
\hline
\end{tabular}

aDAD: diethyl azodicarboxylate. 
${ }^{1} \mathrm{H}$ NMR spectra of cardanol and two DEAD derivatives made via conventional heat (samples C-0, C-1, and C-2) are given in Figure S1 (Supplementary Information section). The spectra from corresponding microwave reactions are similar. The assignments of the ${ }^{1} \mathrm{H}$ spectrum of cardanol have been previously made; ${ }^{18,21}$ the assignments for cardanol triene are annotated in Figure S1a. Note that the peaks at 6.6-7.3 ppm correspond to the phenyl ring protons and the peaks 5.0-6.0 ppm correspond to olefins. After the DEAD reaction (Figures $\mathrm{S} 1 \mathrm{~b}$ and $\mathrm{S} 1 \mathrm{c}$ ), the ethyl group on DEAD can be found at $4.3 \mathrm{ppm}\left(\mathrm{CH}_{2}\right)$ and $1.4 \mathrm{ppm}\left(\mathrm{CH}_{3}\right)$. Furthermore, the phenolic proton is found at 7.2-7.5 ppm. The H-4 on the ring is shifted upfield so that $\mathrm{H}-2, \mathrm{H}-4$, and H-6 all show up as one peak at $6.7 \mathrm{ppm}$ in Figures S1b and S1c (Supplementary Information section). These assignments are consistent with observed peak ratios (1:1:3) for the peaks at 7.3, 7.0 and 6.7 ppm in Figure S1c.

The most interesting spectral change for the DEAD reaction occurs with olefins. It seems that the olefin reactivity roughly follows this trend: triene $>$ diene $>$ mono-ene. Thus, the chain end olefins (H-21 and $\mathrm{H}-20$, from the triene) that appear at 5.1 and $5.8 \mathrm{ppm}$, respectively, decrease in intensity upon reaction with DEAD. For sample C-1, the diene peaks (multiplets at 5.3-5.5 ppm) also decrease in intensity but at a somewhat slower rate (Figure S1b). For sample C-2, diene and chain-end olefins are mostly reacted; however, the mono-ene peak (at $5.3 \mathrm{ppm}$ ) appears unreacted (Figure S1c). Because the hydrazino-ester products can have geometric isomerism (cis and trans) and positional isomerism (with DEAD reacting at different allylic positions), the olefins peaks from the reaction products are broad. If we assume that the major reaction products from diene and triene have conjugated structures, tentative assignments of ${ }^{1} \mathrm{H}$ peaks can be made, where the olefinic $\beta$, $\gamma, \delta$, and $\varepsilon$ protons are found at 5.6, 6.4, 5.9, and $5.4 \mathrm{ppm}$, respectively (Figure 2). More detailed ${ }^{1} \mathrm{H}$ assignments are provided in Figure S2 (Supplementary Information section).

For confirmation, the ${ }^{13} \mathrm{C}$ NMR spectra (105-175 ppm region) for the same three samples $\mathrm{C}-0, \mathrm{C}-1$, and $\mathrm{C}-2$ are shown in Figure S3 in the Supplementary Information section. From ${ }^{13} \mathrm{C}$ chemical shift rules, ${ }^{27}$ the assignments for cardanol have been tentatively assigned in Figure S3a, where phenolic carbons are labelled as $P_{x}$, triene as $T_{x}$, diene as $D_{x}$, and mono-ene as $M_{x}$, where $x=$ carbon number. In sample C-1 (partial addition of DEAD to cardanol), the spectrum (Figure $\mathrm{S} 3 \mathrm{~b}$ ) indicates that the triene peaks decrease about $60 \%$ in intensities, dienes decrease about $50 \%$, and mono-ene stays unchanged. In sample C-2 (further addition of DEAD), the spectrum (Figure S3c) shows little or no triene and diene, but the mono-ene intensity remains the same. In the same sample, there are broad peaks in the 127-135 ppm region, due to the ene reaction products. Because of the possibilities of geometric and positional isomerism, the detailed assignments of these broad peaks are difficult. Tentatively, we attribute the $\beta$ carbon in the conjugated structure to about 129-131 ppm, $\gamma$ and $\delta$ carbons about 127-128 ppm, and $\varepsilon$ carbon around 133 ppm (Figure 2).

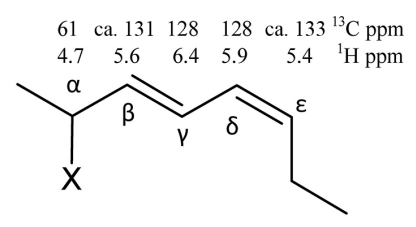

Figure 2. Tentative assignments for ${ }^{1} \mathrm{H}$ and ${ }^{13} \mathrm{C}$ peaks for the conjugated products of cardanol-DEAD reaction, where $\mathrm{X}$ denotes the attachment of the DEAD residue.

The reaction products described in this work are cardanol-aza-dicarboxylate esters. It is of interest to note that the mixture of cardanol and DEAD increases in viscosity upon heating. Table 2 gives the viscosities of cardanol and a cardanol-DEAD reaction mixture as a function of time at $70{ }^{\circ} \mathrm{C}$. By itself, cardanol after 5 hours of heating has a viscosity of $0.3 \mathrm{~Pa}$ s. When cardanolDEAD are mixed together and heated, the viscosity increases steadily with time. The viscosity increase is likely due to the Diels-Alder reaction between the olefin and the diene on the alkenyl moiety of cardanolaza-dicarboxylate ester, leading to self-condensation (Scheme 2). Such self-curing and self-thickening may be of interest and may perhaps be of value as an eco-friendly alternative to petroleum-based thickening, cement, or adhesive systems.

Table 2. Zero-shear viscosity of cardanol and DEAD mixture as a function of time at $70{ }^{\circ} \mathrm{C}$ without a solvent ${ }^{\mathrm{a}}$

\begin{tabular}{lccc}
\hline Sample & $\begin{array}{c}\text { DEAD } \\
\text { weight ratio }\end{array}$ & $\begin{array}{c}\text { Reaction } \\
\text { time / h }\end{array}$ & Viscosity / (Pa s) \\
\hline C-3 & 0 & 5 & 0.3 \\
C-4 & 0.69 & 1 & 22.8 \\
C-5 & 0.69 & 3 & 27.4 \\
C-6 & 0.69 & 5 & 36.7 \\
C-7 & 0.69 & 18 & 63.9 \\
\hline
\end{tabular}

The dependence of the viscosities $(\eta)$ on reaction time $(t)$ for the four DEAD/cardanol samples can be described (correlation coefficient $0.9932)$ by the linear equation: $\eta=21.63+2.38(\mathrm{t})$; ${ }^{b} \mathrm{DEAD}$ : diethyl azodicarboxylate.

In addition to being a viscosifier, the new material may function as a stabilizer and dye dispersant for oil-based lubricants, cosmetics, and personal care formulations. 


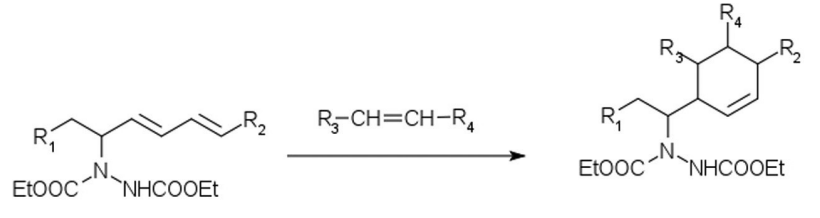

Scheme 2. An example of a Diels-Alder self-condensation reaction for cardanol-DEAD adduct.

Furthermore, this material may be a useful synthon for the preparation of further derivatives. For example, it is known that the hydrazine-ester can undergo alkaline hydrolysis to produce a hydrazine derivative. ${ }^{28}$ Hydrazine can in turn be converted to the amine functionality. ${ }^{29,30}$ Note that in this reaction the olefins in cardanol are shifted in position and not destroyed. Thus, various modification reactions, such as epoxidation and polymerization, reported in the literature for cardanol,,$^{3-8}$ can potentially be carried out on the cardanol-DEAD adduct.

\section{Conclusions}

The reaction described in this work is a rather facile method to introduce electrophilic nitrogen onto the cardanol structure. We have shown in this work that ene reaction between cardanol and DEAD is feasible without a catalyst with conventional heat or in a microwave oven. In the latter case, the reaction can be completed in about 5 minutes, thereby saving time and energy. The reaction product, cardanol-aza-dicarboxylate ester, may be a useful ingredient for commercial formulations. Already it has been shown that the polymer derived from this reaction can increase in viscosity with time and may perhaps serve as a biobased thickener in adhesive, cement, or caulking formulations.

\section{Supplementary Information}

Supplementary information (Figures S1, S2, S3) is available free of charge at http://jbcs.org.br as PDF file.

\section{Acknowledgments}

The authors thank A. J. Thomas for viscosity measurements, Karl Vermillion for NMR data, and Prof Omar El Seoud (University of São Paulo) for helpful suggestions. Atanu Biswas and Roselayne Ferro Furtado thank CNPq (process No. 405506/2013-9) for the support of this work through the Science without Borders program. Mention of trade names or commercial products in this publication is solely for the purpose of providing specific information and does not imply recommendation or endorsement by the U.S. Department of Agriculture. USDA is an equal opportunity provider and employer.

\section{References}

1. Voirin, C.; Caillol, S.; Sadavarte, N. V.; Tawade, B. V.; Boutevin, B.; Wadgaonkar, P. P.; Polym. Chem. 2014, 5, 3142.

2. Velmurugan, A.; Loganathan, M.; World Acad. Sci. Eng. Technol. 2011, 58, 889.

3. Yadav, R.; Srivastava, D.; Eur. Polym. J. 2009, 45, 946.

4. Chuayjuljit, S.; Rattanametangkool, P.; Potiyaraj; J. Appl. Polym. Sci. 2007, 104, 1997.

5. Roy, D.; Basu, P. K.; Raghunathan, P.; Eswaran, S. V.; J. Appl. Polym. Sci. 2003, 89, 1959.

6. Rao, B. S.; Palanisamy, A.; Eur. Polym. J. 2013, 49, 2365.

7. Huang, K.; Zhang, Y.; Li, M.; Lian, J.; Yang, X.; Xia, J.; Prog. Org. Coat. 2012, 74, 240.

8. Sultania, M.; Rai, J.; Srivastava, D.; Mater. Chem. Phys. 2012, 132, 180.

9. Ravichandran, S.; Bouldin, R. M.; Kumar, J.; Nagarajan, R.; J. Cleaner Prod. 2011, 19, 454.

10. Calo, E.; Maffezzoli, A.; Mele, G.; Martina, F.; Mazzetto, S. E.; Tarzia, A.; Stifani, C.; Green Chem. 2007, 9, 754.

11. Tan, T. T. M.; Polym. Int. 1996, 41, 13.

12. Bhunia, H. P.; Jana, R. N.; Basak, A.; Lenka, S.; Nando, G. B.; J. Polym. Sci., Part A: Polym. Chem. 1998, 36, 391.

13. Bhunia, H. P.; Nando, G. B.; Chaki, T. K.; Basak, A.; Lenka, S.; Nayak, P. L.; Eur. Polym. J. 1999, 35, 1381.

14. Suresh, K. I.; Kishanprasad, V. S.; Ind. Eng. Chem. Res. 2005 , 44, 4504.

15. Chen, Z.; Chisholm, B. J.; Webster, D. C.; Zhang, Y.; Patel, S.; Prog. Org. Coat. 2009, 65, 246.

16. Kanehashi, S.; Yokoyama, K.; Masuda, R.; Kidesaki, T.; Nagai, K.; Miyakoshi, T.; J. Appl. Polym. Sci. 2013, 130, 2468.

17. Sandrino, B.; Clemente, C. D. S.; Oliveira, T. M. B. F.; Ribeiro, F. W. P.; Pavinatto, F. J.; Mazzetto, S. E.; de Lima-Neto, P.; Correia, A. N.; Pessoa, C.A.; Wohnrath, K.; Colloids Surf., A 2013, 425, 68.

18. Darroman, E.; Durand, N.; Boutevin, B.; Caillol, S.; Prog. Org. Coat. 2015, 83, 47.

19. Bai, W.; Xiao, X.; Chen, Q.; Xu, Y.; Zheng, S.; Lin, J.; Prog. Org. Coat. 2012, 75, 184.

20. Mi, Z.; Nie, X.; Liu, Z.; Wang, Y.; J. Bioprocess Eng. Biorefinery 2012, 1, 202.

21. Chen, J.; Nie, X.; Liu, Z.; Mi, Z.; Zhou, Y.; ACS Sustainable Chem. Eng. 2015, 3, 1164.

22. Oppolzer, W.; Snieckus, V.; Angew. Chem., Int. Ed. Engl. 1987, 17,476 .

23. Mikami, K.; Shimizu, M.; Chem. Rev. 1992, 92, 1021

24. Nahm, S. H.; Cheng, H. N.; J. Org. Chem. 1986, 51, 5093.

25. Biswas, A.; Sharma, B. K.; Willet, J. L.; Vermillion, K.; Erhan, S. Z.; Cheng, H. N.; Green Chem. 2007, 9, 85. 
26. Biswas, A.; Sharma, B. K.; Willett, J. L.; Erhan, S. Z.; Cheng, H. N.; Green Chem. 2008, 10, 290.

27. Cheng, H. N.; Kasehagen, L. J.; Anal. Chim. Acta 1994, 285, 223.

28. Biswas, A.; Shogren, R. L.; Willett, J. L.; Erhan, S. Z.; Cheng, H. N.; ACS Symp. Ser. 2008, 999, 76.
29. Ren, F.; Zhang, Y.; Hu, L.; Luo, M.; ARKIVOC 2013, 3, 165.

30. Zhang, Y.; Tang, Q.; Luo, M.; Org. Biomol. Chem. 2011, 9 , 4977.

Submitted: September 30, 2015 Published online: January 14, 2016 